\title{
Revisão sistemática: Intersecções entre design thinking e equipe muldisciplinar no processo ensino- aprendizagem
}

Systematic review: Intersections between design thinking and multidisciplinary teams in the teaching-learning process

MACHADO, Andréia de bem

Universidade Federal de Santa Catarina - UFSC I andreiadebem@gmail.com

GUERDT, Elma Mendes

Secretaria de Educação I eguedert65@yahoo.com.br

FIALHO, Francisco Antonio

Universidade Federal de Santa Catarina - UFSC I fapfialho@gmail.com

\begin{abstract}
Resumo
A inovação esta ímbuida na sociedade contemporânea e traz novas metodologias ao fazer pedagógico influenciada pela lente do design thinking. Assim, o objetivo deste artigo é mapear as intersecções entre design thinking e equipe muldisciplinar no processo de ensino-aprendizagem. Para tanto, realizou-se uma busca sistemática na base de dados Scopus. Como resultado, identificou-se que a pesquisa emerge nos campos diferenciados do saber.
\end{abstract}

Palavras-chave: Design thinking. Equipe muldisciplinar. Processo ensino-aprendizagem.

\section{Abstract}

Innovation is ubiquitous in the contemporary society and brings new methodologies to the pedagogical practice, which is influenced by the lenses of design thinking. Thus, the purpose of this paper is to map the intersections between design thinking and multidisciplinary teams in the teaching-learning process. To do so, a systematic review was performed in the Scopus database. Results show that research emerges from a variety of fields of knowledge.

Keywords: Design thinking. Multidisciplinary team. Teaching-learning process. 


\section{INTRODUC̣ÃO}

A formação dos profissionais, ao longo dos anos, foi pautada em metodologias tradicionais, influenciadas por modelos fragmentados, que compartimentam o conhecimento em campos altamente especializados. Isso tornou-se visível nas Instituições de ensino superior, com a subdivisão dessa instituição em departamentos, áreas, campos e cursos, estes em períodos modelados por disciplinas estagnadas. Nesse contexto, o processo de ensino restringiu-se à repetição e reprodução do conhecimento e, assim, o professor passa a ser um transmissor de conteúdos e o estudante, um repetidor de conteúdos. Este, por sua vez, assume uma postura passiva e reprodutora, sem criticar ou refletir sobre o que está sendo abordado pelo educador.

$\mathrm{Na}$ sociedade atual, baseado no conhecimento, imersa em tecnologias inovadoras atreladas aos meios de comunicação, são influenciadas ao uso de novas ferramentas no fazer pedagógico, trazendo processos inovadores. Uma das ferramentas que têm demostrado eficácia na busca por inovação no processo de ensino adotado pelos professores é o design thinking. Segundo Vianna et al. (2012), o design thinking está atrelado à forma de ver as coisas e resolver os problemas, pois utiliza-se de um tipo de raciocínio pouco convencional no meio acadêmico, o pensamento abdutivo, que é um processo participativo para formar hipóteses explicativas. Os autores destacam que "é pensando de maneira abdutiva que o designer constantemente desafia seus padrões, fazendo e desfazendo conjecturas, e transformando-as em oportunidades para a inovação" (VIANNA et al., 2012, p. 14).

O design thinking é usado no processo de ensino aprendizagem com ferramentas para as equipes multidisciplinares inovarem no seu fazer pedagógico no contexto educacional. Compreendo equipe muldisciplinar como

[...] um grupo de indivíduos com contributos distintos, com uma metodologia compartilhada frente a um objetivo comum, cada membro da equipe assume claramente as suas próprias funções, assim como os interesses comuns do coletivo, e todos os membros compartilham as suas responsabilidades e resultados. (ZURRO; FERREROX; BAS, 1991, p. 29).

De acordo com Bento (2007) a composição de uma equipe multidisciplinar é realizada por profissionais de diferentes áreas, ou seja, com formações acadêmicas diversas e que trabalham em prol de um único objetivo. A tarefa multiprofissional implica em responsabilidade, sendo que cada profissional realiza o seu trabalho em consonância com o seu grupo, de maneira que a equipe cresça, principalmente, na qualidade dos serviços prestados. 
Com base nessa contextualização, o objetivo deste estudo é mapear as intersecções entre design thinking equipe multidisciplinar no processo de ensino-aprendizagem. Para tanto, o artigo está planejado em cinco seções. A primeira, anteriormente explicitada, intitula-se "Introdução". Na segunda seção, apresentam-se os procedimentos da pesquisa. Na terceira seção, mostra-se de modo detalhado o resultado bibliométrico de pesquisa, baseado no cenário das publicações científicas da área. Na quarta seção, são explicitadas as considerações finais. Por último, na quinta seção, elencam-se as referências utilizadas.

\section{CAMINHO METODOLÓGICO}

Com o intuito de atender a essa problemática da pesquisa deste estudo, trabalhou-se a partir da metodologia exploratório-descritiva, com o objetivo de delinear o tema e aumentar a familiaridade dos pesquisadores com o fato, contribuindo para ampliar os conceitos inerentes ao tema em estudo (MARCONI; LAKATOS, 2010).

Como método de pesquisa da literatura, utilizou-se a busca sistemática em uma base de dados on-line, seguida de uma análise bibliométrica dos resultados. A bibliometria é uma metodologia oriunda das ciências da informação que, com base em análise quantitativa, permite mapear publicações a partir de registros bibliográficos armazenados em bases de dados (FEATHER; STURGES, 2003; SANTOS; KOBASHI, 2009). Desse modo, considera-se que a proposta metodológica definida atende à problemática de pesquisa, orientando a construção de um estudo que esteja alinhado com o seu objetivo geral.

\subsection{Procedimentos Para Coleta de Dados}

Para a análise bibliométrica, o estudo foi elaborado em três etapas distintas: planejamento, coleta de dados e análise de resultados. Essas etapas aconteceram de modo integrado e síncrono para responder à pergunta norteadora da pesquisa: Quais as intersecções entre design thinking e equipe multidisciplinar para o processo de ensino-aprendizagem?

O planejamento iniciou-se no mês de abril de 2018, quando a pesquisa foi efetivada. No escopo do planejamento, foi estipulada como relevante para o domínio da pesquisa a base de dados Scopus (<http://www.scopus.com>), devido a sua importância no meio acadêmico, seu caráter interdisciplinar, sua atualidade e também por ser uma das maiores bases de resumos e referências bibliográficas de literatura científica revisada por pares. 
Considerando-se o problema de pesquisa, delimitaram-se os termos de busca no início da fase 2 - coleta de dados -, e eles foram definidos como "'design thinking' and "interdisciplinary team". Como princípio básico para a busca, optouse pela utilização dos termos nos campos "Title", "Abstract" e "Keyword", sem restrição temporal, de idioma ou outra qualquer que possa limitar o resultado. Essa fase recuperou um total de 32 trabalhos indexados, com o primeiro registro datado de 2007 e o último de 2016.

$\mathrm{Na}$ terceira fase, de análise de resultados, identificou-se que os trabalhos foram escritos por 51 autores, vinculados a 30 instituições. Foram utilizadas 151 palavras-chave para identificar e indexar as publicações, que se apresentam distribuídas em oito áreas do conhecimento. $O$ quadro 1 apresenta o resultado da coleta de dados numa análise bibliométrica geral dos resultados obtidos na base de dados Scopus.

Quadro 1 - Bibliometria da intersecção entre design thinking e equipe muldisciplinar.

\begin{tabular}{ll}
\hline Base de dados & Scopus \\
\hline Termos de busca & design thinking and interdisciplinary team \\
\hline Campos de busca & Title, Abstract, Keyword \\
\hline Conferência & 12 \\
\hline Capítulo de Livro & 1 \\
\hline Artigos & 5 \\
\hline Autores & 51 \\
\hline Instituições & 30 \\
\hline Países & 17 \\
\hline Palavras-chave & 121 \\
\hline Áreas do conhecimento & 9 \\
\hline
\end{tabular}

Fonte: Elaborado pelos autores (2018).

O universo de 18 trabalhos científicos corresponde a artigos que compõem a amostra para uma análise bibliométrica geral das publicações. As áreas compreendidas são: Ciência da Computação, Engenharia, Ciências Sociais, Artes e Humanidades, Negócios, Gestão e Contabilidade, Matemática, Economia, Econometria e Finanças, Remédio e Enfermagem. Os trabalhos foram publicados entre 2010 e 2016, o que permite tecer o estado da arte do tema a partir da base de dados consultada. Sendo que no ano de 2017 não houve publicação.

Para a apreciação dos resultados de maneira mais aprofundada na análise bibliométrica, fez-se a exportação dos dados para um software de gerenciamento bibliográfico denominado EndNoteWeb. Na sequência, organizou-se uma planilha de dados eletrônica com as informações definidas como relevantes para o estudo: distribuição temporal; principais autores, instituições e países; tipo de publicação na área; principais palavras-chave; trabalhos mais referenciados. 


\section{APRESENTAC̣ÃO DE DADOS E DISCUSSÕES}

Inicialmente, analisou-se a distribuição temporal dos trabalhos, o que permitiu identificar que a primeira publicação está datada de 2007, com um artigo nesse ano. E nos anos de 2008, 2009 e 2010 com um artigo publicado em cada um desses anos. Já em 2011, teve-se um aumento de ocorrências, com a publicação de dois artigos. Em 2012, não tivemos publicações, em 2013, com uma publicação. Em 2014, houve um aumento no número de artigos; quatro foram publicados. No ano seguinte, em 2015, ocorreu uma diminuição com publicação de três artigos. Em 2016, houve um decréscimo novamente, com a publicação de dois artigos. Essa frequência mostra a descontinuidade e a carência de pesquisas na área. Para melhor visualizar esses dados, elaborou-se o gráfico 1.

Gráfico 1 - Distribuição dos trabalhos publicados por ano.

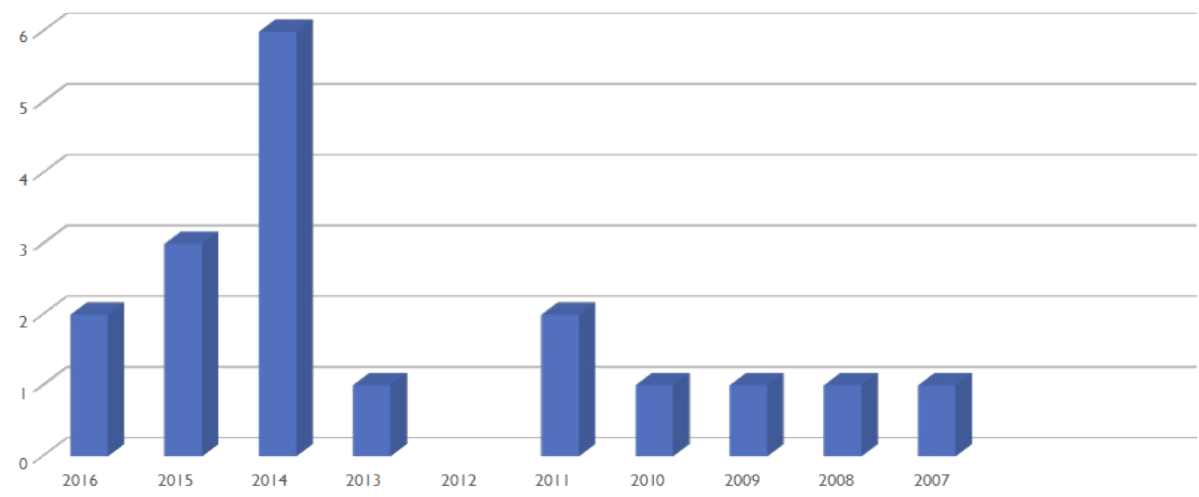

Fonte: Elaborado pelos autores (2017).

Percebe-se que as pesquisas na área podem ser consideradas incipientes, pois apresentam-se, segundo análise quantitativa, em pequena proporção. Infere-se, a partir desse olhar, a necessidade de ampliação das pesquisas relacionadas à temática design thinking e equipe muldisciplinar no processo de ensino-aprendizagem, para que seja possível a exploração de novas metodologias de ensino, as quais permitam a formação de indivíduos críticos que integrem a sociedade contemporânea - uma sociedade do conhecimento. Seguindo-se essa análise, destacam-se no último ano, 2016, duas pesquisas indexadas, como apresentado no quadro 2. 
Quadro 2 - Ocorrências de design thinking na base de dados Scopus em 2016.

\begin{tabular}{|c|c|c|c|}
\hline Autor & Tipo de Publicação & $\begin{array}{l}\text { Título original da } \\
\text { Publicação }\end{array}$ & Resumo do artigo \\
\hline $\begin{array}{l}\text { Roberto, R.A., Da } \\
\text { Silva, M.M.O., } \\
\text { Freitas, D.Q. r, Lima, } \\
\text { Y.C.C.A., Silva, V.E.M, } \\
\text { Araujo, C.C., Teixeira, } \\
\text { J.M.X.N., Teichrieb, } \\
\text { V.a }\end{array}$ & Conferência de papel & $\begin{array}{l}\text { Voxar puzzle motion: } \\
\text { An innovative ar } \\
\text { application proposed } \\
\text { using design } \\
\text { techniques }\end{array}$ & $\begin{array}{l}\text { O trabalho discute } \\
\text { a criação de uma } \\
\text { plataforma que } \\
\text { foi projetada para } \\
\text { promover a educação } \\
\text { das crianças de uma } \\
\text { forma divertida. }\end{array}$ \\
\hline $\begin{array}{l}\text { Signoretti, A.a, } \\
\text { Martins, A.I.b, } \\
\text { Rodrigues, M.c, } \\
\text { Campos, A.M.C.d, } \\
\text { Teixeira, A. }\end{array}$ & Conferência de papel & $\begin{array}{l}\text { Services \& Products } \\
\text { Gamified Design } \\
\text { (SPGD) a methodology } \\
\text { for game thinking } \\
\text { design }\end{array}$ & $\begin{array}{l}\text { A pesquisa propõe } \\
\text { uma metodologia } \\
\text { denominada Serviços } \\
\text { e Produtos com } \\
\text { Design Gamer } \\
\text { (SPGD), que pode ser } \\
\text { usada para projetar } \\
\text { qualquer serviço ou } \\
\text { produto centrado no } \\
\text { usuário. }\end{array}$ \\
\hline
\end{tabular}

Fonte: Elaborado pelos autores (2018).

Observa-se, no quadro 2, apresenta dois trabalhos onde explicita que o design thinking está intersectado com equipe multidisciplinares, contribuindo no processo ensino aprendizagem para a construção e reconstrução significativas de novos conhecimentos. Os artigos publicados também explicitam abordagens pedagógicas adequadas e ferramentas para melhorar o ensino que inovam a aprendizagem dos alunos, como a aprendizagem baseada em problemas e a aprendizagem baseada em projetos.

A partir de um olhar sistêmico e direcionado aos 18 trabalhos localizados, verifica-se uma variada lista de países que se destacam. Os Estados Unidos são o país com maior destaque, com $22 \%$ das publicações totais, ou seja, cinco artigos. Em segundo lugar, tem-se a Países baixo, com 13\% das ocorrências, ou três trabalhos. Em terceiro lugar, estão Brasil, com 9\%, ou seja, dois artigos, que permite evidenciar-se que há uma carência de trabalhos nessa área no cenário nacional. $O$ gráfico 2 demonstra a relação entre países e publicações indexadas na área segundo a base de dados Scopus.

Gráfico 2 - Distribuição das pesquisas por países.

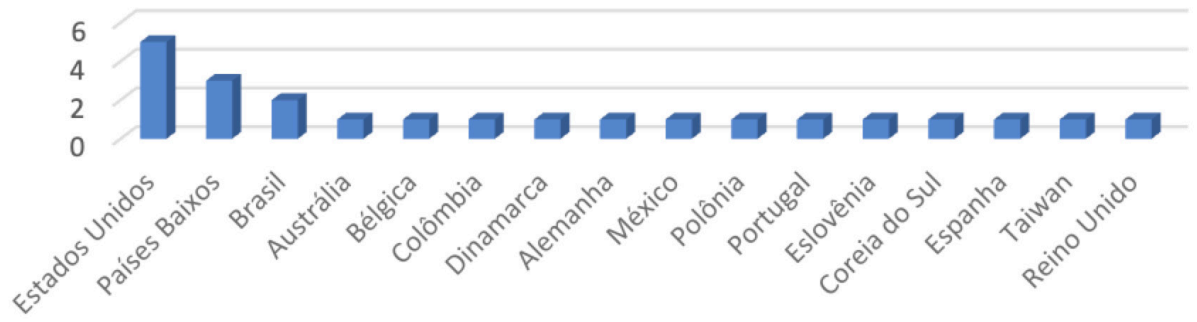

Fonte: Elaborado pelos autores (2018). 
Outra análise quantitativa realizada a partir de um olhar bibliométrico está relacionada ao número de autores que aparecem no resultado da busca. A pequena quantidade de pesquisadores permite afirmar que não existem destaques na área, mas que ela ainda está em construção e desenvolvimento e que os especialistas que nela atuam apresentam-se em número variado de acordo com o país de origem. Observa-se que os 51 autores, cada um publicou um artigo cada um.

Segundo o levantamento geral, foi possível analisar-se ainda as áreas de concentração dos artigos, que se situam nos seguintes campos do conhecimento: Ciência da Computação, Engenharia, Ciências Sociais, Artes e Humanidades, Negócios, Gestão e Contabilidade, Matemática, Economia, Econometria e Finanças, Remédio e Enfermagem., conforme ilustra o gráfico 3.

Gráfico 3 - Áreas do conhecimento das publicações localizadas.

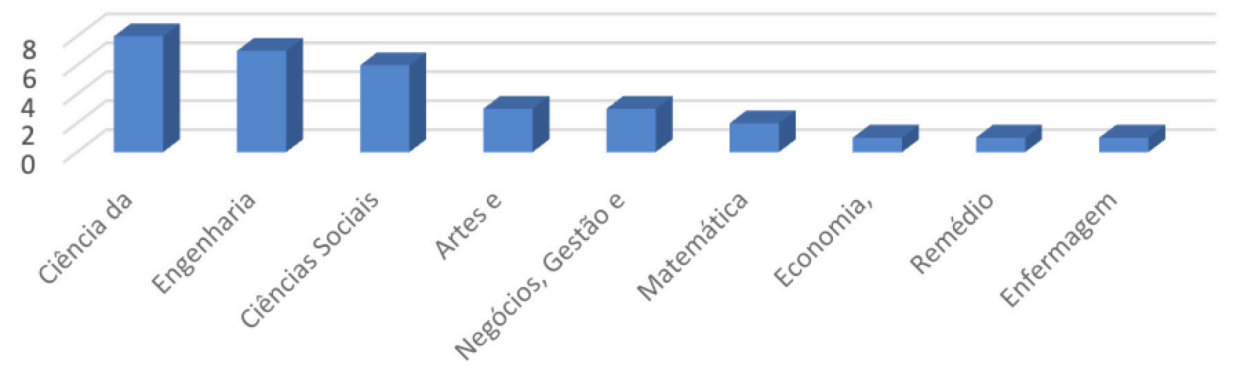

Fonte: Elaborado pelos autores (2018).

Verifica-se que a maior concentração de publicações está na área de Ciência da Computação, com 25\% dos trabalhos, seguida da área de Engenharia, com 22\%, e de Ciências Sociais, com 19\%.

Outra análise realizada a partir da análise bibliométrica, com base no grupo de trabalhos recuperados na base de dados Scopus, foram as palavraschave adotadas, as quais se sintetizam em 151termos. O destaque ficou para "Design de produto", com 10 ocorrências. Em seguida, pode-se ver as seguintes palavras-chave (entre parênteses, a quantidade de ocorrências): Equipes Interdisciplinares (7); desenhar (6); design Thinking (4); Currículos (3); Educação (3); Educação de Engenharia (3); Humano (3); Estudantes (3); Design Centrado no Usuário (3); E-learning (2); Interação Humano-Computador (2); Humanos (2); Métodos Iterativos(2); Ensino (2). As demais ocorrências não foram consideradas neste artigo, pois aparecem com frequência considerada baixa: apenas uma vez. Na figura 1, destacam-se as ocorrências mais frequentes das palavras-chave. 
Figura 1 - Palavras-chave que intersectam a pesquisa sobre design thinking e equipe muldisciplinar

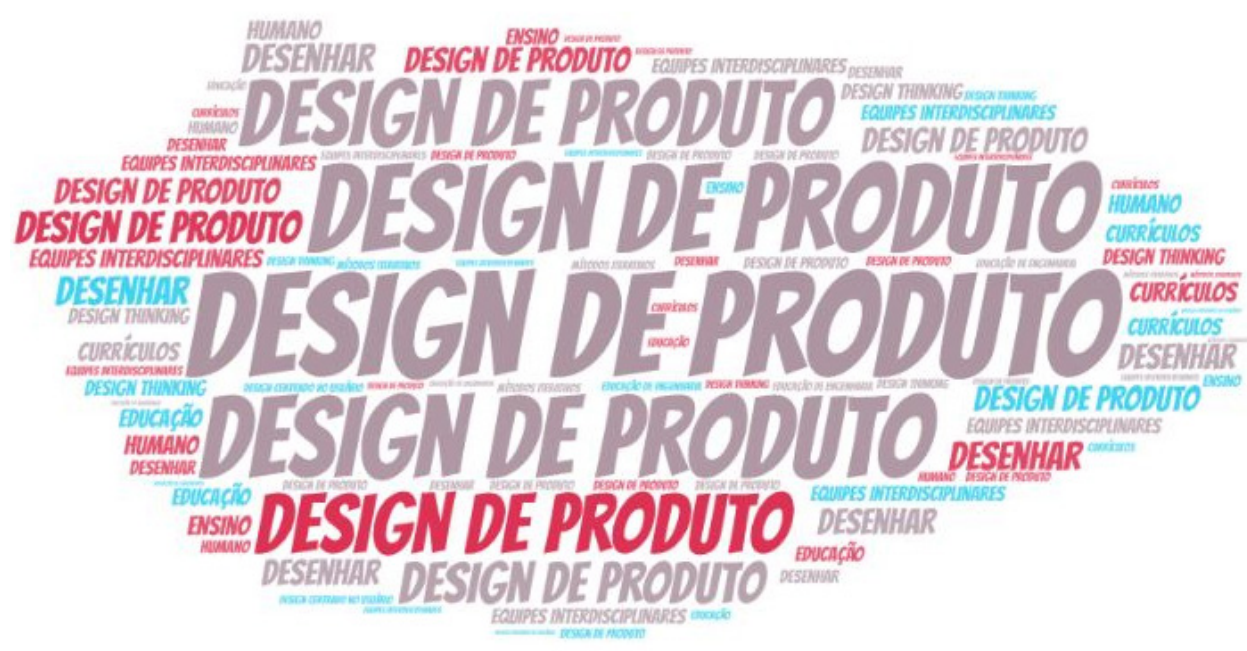

Fonte: Elaborada pelas autoras (2018).

$\mathrm{Na}$ análise das palavras-chave, percebe-se que o debate sobre a intersecção entre design thinking e equipe muldiscipinar congrega questões relacionadas à educação, com destaque para os temas "currículo", "desenho", "ensino" e "design de produto". Isso enfatiza uma proposta ativa de construção do conhecimento, caracterizando a exigência de novas metodologias no fazer pedagógico.

Por fim, numa análise de cunho qualitativo, percebeu-se que a discussão envolve ainda a preocupação com novas metodologias que possam ser utilizadas na equipe muldisciplinar no processo ensino-aprendizagem, trazendo à tona questões como ensinar com a lente do design. Esse fato se constitui numa nova forma de fazer educação que não se limita a conteúdos científicos nem a práticas tradicionais de ensino. Ainda permeiam o debate a evolução das tecnologias de comunicação digital, num processo amplo que envolve aluno, professores, instituição de ensino, família e sociedade.

\section{CONSIDERAC̄ÕES FINAIS}

Tratar do tema "design thinking e equipe muldisciplinar" implica debater aspectos relacionados ao processo didático-pedagógico de ensinar e aprender, tópicos estes atrelados a ferramentas inovadoras que formem um sujeito crítico e autônomo. Nesse contexto, destaca-se a preocupação com o trabalho na área educacional em busca de qualidade no atendimento e na prestação de serviços. Além disso, o uso de ferramentas inovadoras no processo de ensinoaprendizagem promove a interação, pois exige que a abordagem seja centrada no aluno e contemple várias estratégias de ensino que adotem a lente do design para atender aos diferentes tipos de aprendizagem. 
O mapeamento científico da produção relacionada ao tema "design thinking na equipe muldisciplinar no processo ensino-aprendizagem", feito na base de dados Scopus, permitiu uma análise bibliométrica que descrevesse as principais discussões da contemporaneidade e a interseção entre as áreas. Como resultado, identificou-se que a pesquisa emerge no campo multidisciplinar, intersectando as discussões nos campos: Ciência da Computação, Engenharia, Ciências Sociais, Artes e Humanidades, Negócios, Gestão e Contabilidade, Matemática, Economia, Econometria e Finanças, Remédio e Enfermagem.

Finalmente, evidenciou-se que as discussões sobre design thinking e equipe muldisciplinar carecem de estudos e compartilhamento de boas práticas já realizadas. Sugere-se, assim, que estudos nas diferentes áreas sejam analisados e ampliados por um olhar integrado no fazer educacional e que sejam intersectados com planejamentos e práticas educacionais, promovendo ações para educação continuada dos docentes.

\section{REFERÊNCIAS}

BENTO, Ana Maria de O. Percepção da equipe multidisciplinar frente à função do pedagogo numa escola de educação especial. 2007. Disponível em: http:// www.fc.unesp.br/upload/pedagogia/TCC20Ana20Maria20-20Final.pdf. Acesso em: 24 jul. 2018.

FEATHER, John; STURGES, Paul. International encyclopedia of information and library science. Nova lorque: Routledge, 2003. Disponível em: http://mlisuok. weebly.com/uploads/2/6/9/0/26907671/international_encyclopedia_of_ information_ind_librrary_science.pdf. Acesso em: 21 maio 2018.

MARCONI, Marina de Andrade; LAKATOS, Eva M. Fundamentos de metodologia científica. São Paulo: Atlas, 2010.

ROBERTO, Rafael A.; SILVA, Manoela M. O.; FREITAS, Daniel Q.; LIMA, Yvonne, C. C. A.; SILVA, Vinicius E. M; ARAÚJO, Cristiano C.; TEIXEIRA, João Marcelo. Voxar puzzle motion: an innovative ar application proposed using design techniques. In: IEEE VIRTUAL REALITY WORKSHOP ON K-12 EMBODIED LEARNING THROUGH VIRTUAL \& AUGMENTED REALITY (KELVAR), 2016, Greenville. Proceedings [...]. Greenville, SC, USA: IEEE, 2016. p. 11-16. DOI: 10.1109/KELVAR.2016.7563676.

SANTOS, Raimundo Nonato Macedo; KOBASHI, Nair Yumiko. Bibliometria, cientometria, infometria: conceitos e aplicações. Tendências da Pesquisa Brasileira em Ciência da Informação, Brasília, v. 2, n. 1, p. 155-172, 2009. Disponível em: http://inseer.ibict.br/ancib/index.php/tpbci/article/ viewArticle/21. Acesso em: 12 maio 2018. 
SIGNORETTI, Alberto; MARTINS, Ana I.; RODRIGUES, Mario; CAMPOS, André M. C.; TEIXEIRA, António. Services \& products gamified design (SPGD). In: INTERNATIONAL CONFERENCE ON SOFTWARE DEVELOPMENT AND TECHNOLOGIES FOR ENHANCING ACCESSIBILITY AND FIGHTING INFOEXCLUSION, 7., 2016, Vila Real. Proceedings [...]. Vila Reala, 2016. Disponível em: http://dx.doi.org/10.1145/3019943.3019953. Acesso em: 24 jul. 2018.

VIANNA, Mauricio; VIANNA, Ysmar; ADLER, Isabel; LUCENA, Brenda; RUSSO, Beatriz. Design thinking: inovação em negócios. Rio de Janeiro: MJV, 2012.

ZURRO, Armando Martín; FERREROX, P.; BAS, C. S. A equipe de cuidados de saúde primários: manual de cuidados primários. Lisboa: Farmapress, 1991.

Data de submissão: 2018-07-01

Data de aceite: 2018-09-27 